\title{
The Convergence Rate for Difference Approximations to Mixed Initial Boundary Value Problems
}

\author{
By Bertil Gustafsson
}

\begin{abstract}
The convergence rate for difference approximations to mixed initial boundary value problems for hyperbolic systems is treated. Assuming that the approximation at the boundary has one-order lower accuracy than at inner points, conditions are given such that the overall accuracy of the solution is kept at the higher order.
\end{abstract}

1. Introduction. When dealing with difference approximations to mixed initial boundary value problems, one often has trouble defining the difference operators near the boundaries. As an example, consider the equation $\partial u / \partial t=\partial u / \partial x$, which is well posed in $L^{2}(0, \infty)$ for $0 \leqslant x<\infty, t \geqslant 0$ without any boundary condition given at $x=0$. However, any difference approximation, that uses centered difference operators for approximating $\partial u / \partial x$, breaks down at $x=0$ since no values are defined for $x<0$.

One possibility is to use one-sided operators at the boundaries, another to perform some sort of extrapolation. For various reasons, e.g., stability considerations, one uses methods, which yield one-order lower accuracy than the one used at inner points. The question then is if, despite this fact, the overall accuracy of the solution to the difference approximation can be kept at the higher order. In this paper, conditions are given such that this is the case.

The theory used is the one developed in [1], and it is assumed that the reader is familiar with that paper.

2. Definitions, Assumptions and Main Theorems. We consider a hyperbolic firstorder system of partial differential equations

$$
\partial U(x, t) / \partial t=A \partial U(x, t) / \partial x+B U(x, t)+F(x, t), \quad 0 \leqslant x<\infty, t \geqslant 0,
$$

where $A$ is a diagonal matrix, and

$$
A=\left[\begin{array}{ll}
A^{\mathrm{I}} & 0 \\
0 & A^{\mathrm{II}}
\end{array}\right],
$$

with $A^{\mathrm{I}}$ of order $l \times l, A^{\mathrm{I}}<0$ and $A^{\mathrm{II}}$ of order $(n-l) \times(n-l), A^{\mathrm{II}}(0)>0$. For simplicity, we treat only the constant coefficient case; the technique used in [1] for the

Received February 20, 1974.

AMS (MOS) subject classifications. (1970). Primary 65M10, 65M 15.

Key words and phrases. Difference approximations, initial boundary value problems, hyperbolic systems, convergence rate, stability, boundary conditions. 
stability proofs easily allows the treatment of variable coefficients.

Initial and boundary conditions are

$$
\begin{array}{ll}
U(x, 0)=U^{(0)}(x), & 0 \leqslant x<\infty, \\
U^{\mathrm{I}}(0, t)=S U^{\mathrm{II}}(0, t)+G(t), & t \geqslant 0,
\end{array}
$$

where $U^{\mathrm{I}}, U^{\mathrm{II}}$ correspond to the partition of $A$ and where $S$ is a rectangular matrix.

Using the notation $V_{\nu}(t)=V\left(x_{\nu}, t\right), x_{\nu}=v h, \nu=-r+1,-r+2, \cdots$, the difference scheme has the general form

$$
Q_{-1} V_{\nu}(t+k)=\sum_{\sigma=0}^{s} Q_{\sigma} V_{\nu}(t-\sigma k)+k F_{\nu}(t), \quad \nu=1,2, \cdots
$$

where

$$
Q_{\sigma}=\sum_{j=-r}^{p} A_{j \sigma} E^{j}, \quad E V_{\nu}(t)=V_{\nu+1}(t)
$$

Initial and boundary conditions are

$$
\begin{aligned}
V_{\nu}(\sigma k) & =V_{\nu}^{(\sigma)}, \quad \sigma=0,1, \cdots, s, \quad \nu=-r+1,-r+2, \cdots, \\
V_{\nu}(t+k) & =\sum_{\sigma=-1}^{\dot{\delta}} S_{\sigma}^{(\nu)} V_{1}(t-\sigma k)+G_{\nu}(t), \quad \nu=-r+1,-r+2, \cdots, 0,
\end{aligned}
$$

where $S_{\sigma}^{(\nu)}=\Sigma_{j=0}^{q} C_{j \sigma}^{(\nu)} E^{j}$

The same assumptions on the difference approximation are made as in [1]. In particular, it is assumed that $(2.2 \mathrm{a}),(2.2 \mathrm{c})$ can be solved boundedly for $V(t+k)[1$, Assumption 3.1].

We define difference operators in both the $x$ - and $t$-directions by

$$
\begin{aligned}
& D_{+x} W_{\nu}(t)=\left(W_{\nu+1}(t)-W_{\nu}(t)\right) / h, \\
& D_{+t} W_{\nu}(t)=\left(W_{\nu}(t+k)-W_{\nu}(t)\right) / k .
\end{aligned}
$$

With $|\cdot|$ denoting the Euclidian vector norm, we also define

$$
\begin{gathered}
\|W(t)\|_{x}^{2}=\sum_{\nu=-r+1}^{\infty}\left|W_{\nu}(t)\right|^{2} h, \\
\|W\|_{x, t}^{2}=\sum_{\sigma=0}^{\infty}\|W(\sigma k)\|_{x}^{2} k .
\end{gathered}
$$

Sometimes, we will also use the notation $\|\phi\|_{x}$ for functions $\phi_{\nu}(t)$ which are not primarily defined for $\nu \leqslant 0$. In those cases, the missing values are defined as zeros.

We now make

Assumption 2.1. The order of accuracy is $m$ for (2.2a) and at least $m-1$ for (2.2b), (2.2c), and it is assumed that $m \geqslant 1$.

To be more precise, this means that, for all sufficiently smooth solutions to (2.1), we have for $W=U-V$ : 


$$
Q_{-1} W(t+k)=\sum_{\sigma=0}^{s} Q_{\sigma} W(t-\sigma k)+h^{m+1} d_{\nu}(t)
$$

$$
\begin{gathered}
\nu=1,2, \cdots ; t=s k,(s+1) k, \cdots, \\
W_{\nu}(\sigma k)=h^{\beta} e_{\nu}(\sigma k), \quad \nu=-r+1,-r+2, \cdots ; \sigma=0,1, \cdots, s, \\
W_{\nu}(t+k)=\sum_{\sigma=-1}^{s} S_{\sigma}^{(\nu)} W_{1}(t-\sigma k)+h^{\beta} f_{\nu}(t), \\
\nu=-r+1,-r+2, \cdots, 0 ; t=s k,(s+1) k, \cdots,
\end{gathered}
$$

where $d, e, f$ are bounded functions and $\beta \geqslant m$. (We always assume $k / h=$ const.)

As an example consider the equation $\partial U / \partial t=\partial U / \partial x$ with initial condition $U(x, 0)=e(x)$ and the second-order leap-frog difference approximation $W_{\nu}(t+k)=$ $(k / h)\left(W_{\nu+1}(t)-W_{\nu-1}(t)\right)+W_{\nu}(t-k)$. With $W_{\nu}(0)=e\left(x_{\nu}\right), W_{\nu}(k)$ can be defined by $W_{\nu}(k)=(k / h)\left(W_{\nu+1}(0)-W_{\nu}(0)\right)+W_{\nu}(0)$ which is first-order accurate but locally of second order, which means that $\beta=2$ in $(2.3 \mathrm{~b})$. As boundary condition we could use $W_{0}(t+k)=W_{0}(t)+(k / h)\left(W_{1}(t)-W_{0}(t)\right)$ or $W_{0}(t)=2 W_{1}(t)-W_{2}(t)$, which both are of first-order accuracy according to our definition, i.e., $\beta=2$ in $(2.3 \mathrm{c})$.

Let $u_{\nu}(t)$ be the solution of the discrete Cauchy problem, i.e., the difference scheme (2.2a) with the initial condition (2.2b) defined for $\nu=0, \pm 1, \pm 2, \cdots$. Then we make

Assumption 2.2. The difference approximation is stable for the Cauchy problem, i.e., if $F_{\nu}(t) \equiv 0$, then there are constants $K>0, \alpha_{1} \geqslant 0$ such that

$$
\sum_{\nu=-\infty}^{\infty}\left|u_{\nu}(t)\right|^{2} h \leqslant K e^{\alpha_{1} t} \sum_{\sigma=0}^{s} \sum_{\nu=-\infty}^{\infty}\left|u_{\nu}(\sigma k)\right|^{2} h .
$$

Certain smoothness assumptions on $d, e, f$ are required; these will be specified in the theorems. We also need a certain compatibility between initial and boundary conditions:

Assumption 2.3. The functions $e_{\nu}(t)$ and $f_{\nu}(t)$ are such that

$$
\begin{aligned}
& \left|f_{\nu}(s k)-h^{-m}\left(e_{\nu}(s k+k)-\sum_{\sigma=-1}^{s} S_{\sigma}^{(\nu)} e_{1}(s k-\sigma k)\right)\right| \leqslant O(h), \\
& v=-r+1, \cdots, 0 .
\end{aligned}
$$

Here, $e_{\nu}(s k+k)$ is defined by $Q_{-1} e_{\nu}(s k+k)=\Sigma_{\sigma=0}^{s} Q_{\sigma} e(s k-\sigma k)+h^{m+1} d_{\nu}(s k)$.

Connected with $(2.2 \mathrm{a}, \mathrm{c})$, there is the resolvent equation

$$
\begin{aligned}
\left(Q_{-1}-\sum_{\sigma=0}^{s} z^{-\sigma-1} Q_{\sigma}\right) \hat{w}_{\nu} & =\hat{g}_{\nu}, \quad \nu=1,2, \cdots, \\
\hat{w}_{\nu}-\sum_{\sigma=-1}^{s} z^{-\sigma-1} S_{\sigma}^{(\nu)} \hat{w}_{1}=\hat{g}_{\nu}, & \nu=-r+1, \cdots, 0,
\end{aligned}
$$


which can be rewritten in one-step form

$$
w_{\nu+1}=M w_{\nu}+G_{\nu}, \quad w_{\nu}=\left(\hat{w}_{\nu+p-1}, \cdots, \hat{w}_{\nu-r}\right)^{\prime} .
$$

After making the transformation $y=T w$ as in Sections 8, 9, 10 of [1], the boundary conditions can be written $D^{\mathrm{I}}(z) y_{1}^{\mathrm{I}}+D^{\mathrm{II}}(z) y_{1}^{\mathrm{II}}=g+R(G)$, where $y^{\mathrm{I}}$ is that part of $y$ which lies in $l_{2}(0, \infty)$ for $G \equiv 0,|z|>1$. The properties of $\operatorname{Det}\left(D^{\mathrm{I}}(z)\right)$ for $|z| \geqslant 1$ are crucial for the stability.

We can now state our first theorem:

TheOREM 2.1. Assume that there is a constant $K$ such that the solutions to (2.6) fulfill

$$
\sum_{\nu=-r+1}^{0}\left|\hat{w}_{\nu}\right|^{2} \leqslant K \sum_{\nu=-r+1}^{0}\left|\hat{g}_{\nu}\right|^{2}
$$

for all $|z|$ with $|z|>1$, or, equivalently,

$$
\operatorname{Det}\left(D^{\mathrm{I}}(z)\right) \neq 0 \text { for all }|z| \text { with }|z| \geqslant 1 .
$$

Then there are constants $c_{1}$ and $\alpha_{0}, \alpha_{0} \geqslant 0$ such that

$$
\begin{aligned}
& \frac{\alpha-\alpha_{0}}{1+\alpha k} \sum_{\nu=-r+1}^{0}\left\|e^{-\alpha t} W_{\nu}\right\|_{t}^{2}+\left(\frac{\alpha-\alpha_{0}}{1+\alpha k}\right)^{2}\left\|e^{-\alpha t} W\right\|_{x, t}^{2} \\
& \leqslant c_{1} h^{2 m}\left\{\frac { \alpha - \alpha _ { 0 } } { 1 + \alpha k } \left(\sum_{\nu=-r+1}^{0}\left\|e^{-\alpha t} f_{\nu}\right\|_{t}^{2}+\left(\alpha-\alpha_{1}\right)^{-1}\right.\right. \\
&\left.\cdot \sum_{\sigma=0}^{s}\left[\left(2+\frac{\alpha-\alpha_{0}}{1+\alpha k}\right)\|e(\sigma k)\|_{x}^{2}+\left\|D_{+x} e(\sigma k)\right\|_{x}^{2}\right]\right) \\
&\left.+\left\|e^{-\alpha t} d\right\|_{x, t}^{2}\right\}
\end{aligned}
$$

for all $\alpha$ with $\alpha>\max \left(\alpha_{0}, \alpha_{1}\right) \quad\left(\alpha_{1}\right.$ is defined by (2.4)). Therefore, if the norms in the right-hand side of (2.9) are bounded independently of $h$, the convergence rate is of order $m$. (The proof of Theorem 2.1 is given in the next section.)

It should be noted that, for the constant coefficient case treated here, the constants $\alpha_{0}, \alpha_{1}$ can be taken zero if there is no lower-order term in (1.1). However, the results given here are valid even for variable coefficients since the main theorems used from [1] are proved by the energy method (see also Sections 10, 11 of [2] where the variable coefficient case is treated).

An exponential growth $\left(\alpha_{0}>0\right)$ can also occur for the half-strip problem $0 \leqslant$ $x \leqslant 1, t \geqslant 0$, even with constant coefficients. Also, in this case, the results here are valid, and $\alpha_{0}$ is not necessarily equal to $\alpha_{1}$.

With a slightly stronger smoothness requirement on $d, e, f$, we are able to weaken the condition (2.8) and still find the convergence rate to be of order $m$ :

Theorem 2.2. Assume that $\operatorname{Det}\left(D^{\mathrm{I}}(1)\right) \neq 0$, and that there is a constant $K$ such 
that, for all $z$ with $|z|>1$, the solutions to (2.6) with $\hat{g}_{\nu}=0, \nu=1,2, \cdots$, satisfy

$$
\|\hat{w}\|_{x}^{2} \leqslant K \frac{|z|^{2}}{(|z|-1)^{2}} \sum_{\nu=-r+1}^{0}\left|\hat{g}_{\nu}\right|^{2} h .
$$

Then, there are constants $c_{2}$ and $\alpha_{0}, \alpha_{0} \geqslant 0$ such that

$$
\begin{gathered}
\frac{\alpha-\alpha_{0}}{1+\alpha k} \sum_{\nu=-r+1}^{0}\left\|e^{-\alpha t} W_{\nu}\right\|_{t}^{2}+\left(\frac{\alpha-\alpha_{0}}{1+\alpha k}\right)^{2}\left\|e^{-\alpha t} W\right\|_{x, t}^{2} \\
\leqslant c_{2} h^{2 m}\left\{\frac{\alpha-\alpha_{0}}{1+\alpha k} \sum_{\nu=-r+1}^{0}\left\|e^{-\alpha t} f_{\nu}\right\|_{t}^{2}+\alpha^{-1} \sum_{\nu=-r+1}^{0}\left(\cdot \sum_{\sigma=s}^{\infty}\left|e^{-\sigma k} D_{+t} f_{\nu}(\sigma k)\right|\right)^{2}\right. \\
+\frac{\alpha-\alpha_{0}}{1+\alpha k}\left(\alpha-\alpha_{1}\right)^{-1}\left[\left(\frac{\alpha-\alpha_{0}}{1+\alpha k}+1\right)\left(\sum_{\sigma=0}^{s}\|e(\sigma k)\|_{x}^{2}+\sup _{\tau \geqslant s k}\|d(\tau)\|_{x}^{2}\right)\right. \\
+\alpha^{-1}\left(\alpha-\alpha_{1}\right)^{-2}\left[\sum_{\sigma=0}^{s-1}\left(\left\|D_{+t} e(\sigma k)\right\|_{x}^{2}+\left\|D_{+x} e(\sigma k)\right\|_{x}^{2}+\sup _{\tau \geqslant s k}\left\|D_{+x} d(\tau)\right\|_{x}^{2}\right]\right. \\
\left.\left.+\sup _{\tau \geqslant s k}\left\|D_{+t} d(\tau)\right\|_{x}^{2}+\sup _{\tau \geqslant s k}\left\|D_{+x} D_{+t} d(\tau)\right\|_{x}^{2}\right]\right\}
\end{gathered}
$$

for all $\alpha$ with $\alpha>\max \left(\alpha_{0}, \alpha_{1}\right)$ ( $\alpha_{1}$ again defined by (2.4)): Under the assumption that the norms in the right-hand side of (2.11) are bounded independently of $h$, the convergence rate is again of order $m$. (The proof of Theorem 2.2 is given in the next section.)

\section{Proof of the Theorems.}

Proof of Theorem 2.1. The functions $e_{\nu}(\sigma k)$ in (2.3b) are extended in a smooth way for $\nu \leqslant-r$ such that they are zero for $\nu h \leqslant-1$, say, and such that

$$
\sum_{\nu=-\infty}^{\infty}\left|e_{\nu}(\sigma k)\right|^{2} h \leqslant \text { const }\|e(\sigma k)\|_{x}^{2},
$$

$$
\sum_{\nu=-\infty}^{\infty}\left|D_{+x} e_{\nu}(\sigma k)\right|^{2} h \leqslant \text { const }\left\|D_{+x} e(\sigma k)\right\|_{x}^{2}
$$

for $\sigma=0,1, \cdots, s$.

We define the Cauchy problem

$$
\left.\begin{array}{rl}
Q_{-1} u_{\nu}(t+k) & =\sum_{\sigma=0}^{s} Q_{\sigma} u_{\nu}(t-\sigma k) \\
u_{\nu}(\sigma k) & =h^{m} e_{\nu}(\sigma k), \quad \sigma=0,1, \cdots, s
\end{array}\right\} \quad \nu=0, \pm 1, \cdots,
$$

and the corresponding one for the divided differences $D_{+x} u_{\nu}(t)$. By Assumption 2.2, $\|u(t)\|_{x}$ and $\left\|D_{+x} u(t)\right\|_{x}$ can be estimated, and taking (3.1) into account, we obtain 


$$
\left|u_{\nu}(t)\right|^{2} \leqslant \text { const } e^{\alpha_{1} t} h^{2 m} \sum_{\sigma=0}^{s}\left(\|e(\sigma k)\|_{x}^{2}+\left\|D_{+x} e(\sigma k)\right\|_{x}^{2}\right),
$$

$$
\nu=0, \pm 1, \pm 2, \cdots
$$

The function $v \equiv W-u$ satisfies

$$
\begin{aligned}
Q_{-1} v_{\nu}(t+k) & =\sum_{\sigma=0}^{s} Q_{\sigma} v_{\nu}(t-\sigma k)+h^{m+1} d_{\nu}(t), \quad \nu=1,2, \cdots \\
v_{\nu}(\sigma k) & =0, \quad \sigma=0,1, \cdots ; s ; \quad v=-r+1,-r+2, \cdots \\
v_{\nu}(t+k) & =\sum_{\sigma=-1}^{s} S_{\sigma}^{(\nu)} v_{1}(t-\sigma k)+h^{m} g_{\nu}(t), \quad \nu=-r+1, \cdots, 0,
\end{aligned}
$$

where

$$
h^{m} g_{\nu}(t) \equiv h^{m} f_{\nu}(t)+u_{\nu}(t+k)-\sum_{\sigma=-1}^{s} S_{\sigma}^{(\nu)} u_{1}(t-\sigma k) .
$$

Taking (3.3) into account, we obtain from Theorem 5.1 in [1]

$$
\begin{aligned}
& \frac{\alpha-\alpha_{0}}{1+\alpha k} \sum_{\nu=-r+1}^{0}\left\|e^{-\alpha t} v_{\nu}\right\|_{t}^{2}+\left(\frac{\alpha-\alpha_{0}}{1+\alpha k}\right)^{2}\left\|e^{-\alpha t} v\right\|_{x, t}^{2} \\
& \leqslant \text { const } h^{2 m}\left\{\frac { \alpha - \alpha _ { 0 } } { 1 + \alpha k } \left(\sum_{\nu=-r+1}^{0}\left\|e^{-\alpha t} f_{\nu}\right\|_{t}^{2}+\frac{1}{\alpha-\alpha_{1}}\right.\right. \\
& \left.\left.\sum_{\sigma=0}^{s}\left[\|e(\sigma k)\|_{x}^{2}+\left\|D_{+x} e(\sigma k)\right\|_{x}^{2}\right]\right)+\left\|e^{-\alpha t} d\right\|_{x, t}^{2}\right\} .
\end{aligned}
$$

Combining this inequality with

$$
\left\|e^{-\alpha t} u\right\|_{x, t}^{2} \leqslant \text { const } h^{2 m}\left(\alpha-\alpha_{1}\right)^{-1} \sum_{\sigma=0}^{s}\|e(\sigma k)\|_{x}^{2}
$$

and (3.3), we obtain (2.9), and the theorem is proved.

Proof of Theorem 2.2. As in the above proof, the Cauchy problem is first solved with extended initial functions, but with the term $h^{m+1} d_{\nu}(t)$ added to the right-hand side of (3.2a), where $d_{\nu}(t)$ is extended in the same way as $e_{\nu}(t)$. We then obtain for $\alpha>\alpha_{1}$

We also get

$$
\left\|e^{-\alpha t} u\right\|_{x, t}^{2} \leqslant \text { const } h^{2 m}\left(\alpha-\alpha_{1}\right)^{-1}\left(\sum_{\sigma=0}^{s}\|e(\sigma k)\|_{x}^{2}+\sup _{\tau \geqslant s k}\|d(\tau)\|_{x}^{2}\right) .
$$

$$
\begin{aligned}
\left|u_{\nu}(t)\right|^{2} \leqslant \text { const } e^{\alpha_{1} t} h^{2 m} & \left\{\sum_{\sigma=0}^{s}\left(\|e(\sigma k)\|_{x}^{2}+\left\|D_{+x} e(\sigma k)\right\|_{x}^{2}\right)\right. \\
& \left.+\sup _{s k \leqslant \tau \leqslant t}\|d(\tau)\|_{x}^{2}+\sup _{s k \leqslant \tau \leqslant t}\left\|D_{+x} d(\tau)\right\|_{x}^{2}\right\} .
\end{aligned}
$$


It now remains to estimate the solution $v_{\nu}(t)$ of the problem (3.4) without any inhomogeneous term in (3.4a), but with $g_{\nu}(t)$ in (3.4c) defined by

$$
\begin{aligned}
h^{m} g_{\nu}(t)=h^{m} f_{\nu}(t)-\left\{u_{\nu}(t+k)-\right. & \left.\sum_{\sigma=-1}^{s} S_{\sigma}^{(\nu)} u_{1}(t-\sigma k)\right\}, \\
\nu & =-r+1, \cdots, 0 ; t \geqslant s k .
\end{aligned}
$$

We construct a function $\widetilde{g}_{\nu}(t)$ which, for every fixed $k$, is piecewise differentiable in $t$ :

$$
\tilde{g}_{\nu}(t+\theta k)=(1-\theta) g_{\nu}(t)+\theta g_{\nu}(t+k), \quad t=s k,(s+1) k, \cdots, 0 \leqslant \theta \leqslant 1 .
$$

$\widetilde{v}_{\nu}(t)$ is defined, for all $t \geqslant 0$, as the solution of (3.4) with $g=\tilde{g}$ in (3.4c). We make the variable transformation $\widetilde{w}_{\nu}(t)=e^{-\alpha t} \widetilde{v}_{\nu}(t)$ for $t \geqslant s k$ and define $\widetilde{w}_{\nu}(t) \equiv \widetilde{g}_{\nu}(t) \equiv 0$ for $t<s k$. From our smoothness assumptions, we know that $\widetilde{g_{\nu}}(s k)=O(h)$, and that

$$
\left|\partial \widetilde{g}_{\nu}(t) / \partial t\right| \leqslant \text { const }, \quad-\infty<t<\infty,
$$

independently of $h$. The Eqs. (3.4) are Fourier transformed, and with $z=e^{(\alpha+i \omega) k}$,

$$
\hat{w}_{\nu}(\omega, \alpha)=F w_{\nu}(t), \quad \hat{g}_{\nu}(\omega, \alpha)=h^{m} e^{-i \omega k} F e^{-\alpha(t+k)} \widetilde{g}_{\nu}(t),
$$

Eqs. (2.6) are obtained. We need the following lemma:

Lemma.3.1. Under the assumptions in Section 2, the function $\hat{g}_{\nu}(\omega, \alpha)$ satisfies $\left|\hat{g}_{\nu}(\omega, \alpha)\right|^{2} \leqslant K(\alpha+|\omega|)^{-2}\left\{\left(\sum_{\sigma=s}^{\infty}\left|e^{-\alpha t} D_{+t} f_{\nu}(\sigma k)\right| k\right)^{2}+\left(\alpha-\alpha_{1}\right)^{-1}\right.$

$$
\begin{aligned}
& \cdot\left[\sum_{\sigma=0}^{s-1}\left(\left\|D_{+t} e(\sigma k)\right\|_{x}^{2}+\left\|D_{+x} D_{+t} e(\sigma k)\right\|_{x}^{2}\right)\right. \\
& \left.\left.\quad+\sup _{\tau \leqslant s k}\left\|D_{+t} \cdot d(\tau)\right\|_{x}^{2}+\sup _{\tau \geqslant s k}\left\|D_{+x} D_{+t} d(\tau)\right\|_{x}^{2}\right]\right\},
\end{aligned}
$$

where $K$ is independent of $h, d_{\nu}(t), e_{\nu}(t), f_{\nu}(t)$.

Proof. Considering our Cauchy problem for $u_{\nu}(t)$ and $u_{\nu}(t+k)$, we can immediately estimate $\left\|D_{+t} u(t)\right\|_{x}^{2}$ and $\left\|D_{+x} D_{+t} u(t)\right\|_{x}$. From our smoothness assumptions and the definition of $g_{\nu}(t)$, we therefore get

$$
\left|D_{+t} g_{\nu}(t)\right|^{2} \leqslant\left|D_{+t} f_{\nu}(t)\right|^{2}
$$

$$
\begin{aligned}
+ \text { const } e^{\alpha_{1} t} & \left\{\sum_{\sigma=0}^{s-1}\left(\left\|D_{+t} e(\sigma k)\right\|_{x}^{2}+\left\|D_{+x} D_{+t} e(\sigma k)\right\|_{x}^{2}\right)\right. \\
& \left.+\sup _{s k \leqslant \tau \leqslant t}\left\|D_{+t} d(\tau)\right\|_{x}^{2}+\sup _{s k \leqslant \tau \leqslant t}\left\|D_{+x} D_{+t} d(\tau)\right\|_{x}^{2}\right\} .
\end{aligned}
$$

Using integration by parts, we obtain

$$
h^{-m}\left|\hat{g}_{\nu}(\omega, \alpha)\right| \leqslant \text { const }(\alpha+|\omega|)^{-1} \int_{0}^{\infty}\left|e^{-(\alpha+i \omega) t}\right|\left|\partial \tilde{g}_{\nu}(t) / \partial t\right| d t,
$$

and the lemma follows from (3.8), (3.11). 
The matrix $D^{\mathrm{I}}(z)$ defined in Section 2 has, by assumption, the property $\operatorname{Det}\left(D^{\mathrm{I}}(1)\right) \neq 0$, hence, by continuity, there is a constant $\delta>0$ such that $\operatorname{Det}\left(D^{\mathrm{I}}(z)\right) \neq$ 0 for $|\omega h| \leqslant \delta, z=e^{(\alpha+i \omega) k}, k \leqslant k_{0}, k_{0}>0$. The function $\hat{g}$ is now divided into two parts:

$$
\begin{aligned}
& \hat{g}_{\nu}^{(1)}(\omega, \alpha)= \begin{cases}\hat{g}_{\nu}(\omega, \alpha) & \text { for }|\omega| \leqslant \delta h^{-1}, \\
0 & \text { else, }\end{cases} \\
& \hat{g}_{\nu}^{(2)}(\omega, \alpha)=\hat{g}_{\nu}(\omega, \alpha)-\hat{g}_{\nu}^{(1)}(\omega, \alpha) .
\end{aligned}
$$

Let $\hat{w}^{(1)}, \hat{w}^{(2)}$ be the solutions to (2.6) with the right-hand side of (2.6c) replaced by $\hat{g}_{\nu}^{(1)}, \hat{g}_{\nu}^{(2)}$, respectively, and let $e^{-\alpha t} v^{(i)}$ be the discrete function corresponding to $F^{-1} \hat{w}^{(i)}$. By using Theorems 4.1, 4.2 and 5.1 of [1], we then obtain in a straightforward manner

$$
\frac{\alpha-\alpha_{0}}{1+\alpha k} \sum_{\nu=-r+1}^{0}\left\|e^{-\alpha t} v_{\nu}^{(1)}\right\|_{t}^{2}+\left(\frac{\alpha-\alpha_{0}}{1+\alpha k}\right)^{2}\left\|e^{-\alpha t} v^{(1)}\right\|_{x, t}^{2}
$$

$$
\leqslant \text { const } \frac{\alpha-\alpha_{0}}{1+\alpha k} h^{2 m}\left\{\sum_{\nu=-r+1}^{0}\left\|e^{-\alpha t} f_{\nu}\right\|_{t}^{2}\right.
$$

and

$$
\begin{aligned}
+\frac{1}{\alpha-\alpha_{1}}\left[\sum_{\sigma=0}^{s}\left(\|e(\sigma k)\|_{x}^{2}+\left\|D_{+x} e(\sigma k)\right\|_{x}^{2}\right)\right. \\
\left.\left.+\sup _{t \geqslant s k}\|d(t)\|_{x}^{2}+\sup _{t \geqslant s k} D_{+x} d(t) \|_{x}^{2}\right]\right\}
\end{aligned}
$$

$$
\left(\frac{\alpha-\alpha_{0}}{1+\alpha k}\right)^{2}\left\|e^{-\alpha t} v^{(2)}\right\|_{x, t} \leqslant \text { const } k^{-1} \int_{|\omega|>\delta h^{-1}} \sum_{\nu=-r+1}^{0}\left|\hat{g}_{\nu}^{(2)}\right|^{2} d \omega .
$$

Since $\int_{|\omega|>\delta h^{-1}}(\alpha+|\omega|)^{-2} d \omega \leqslant O(h / \alpha)$, we get from Lemma 3.1

$$
\begin{aligned}
& \left(\frac{\alpha-\alpha_{0}}{1+\alpha k}\right)^{2}\left\|w^{(2)}\right\|_{x, t} \\
& \leqslant \text { const } \alpha^{-1} h^{2 m}\left\{\sum_{\nu=-r+1}^{0}\left(\sum_{\sigma=s}^{\infty}\left|e^{-\sigma k} D_{+t} f_{\nu}(\sigma k)\right| k\right)^{2}\right. \\
& +\left(\alpha-\alpha_{1}\right)^{-2}\left[\sum_{\sigma=0}^{s-1}\left(\left\|D_{+t} e(\sigma k)\right\|_{x}^{2}+\left\|D_{+x} D_{+t} e(\sigma k)\right\|_{x}^{2}\right)\right. \\
& \left.\left.+\sup _{t \geqslant s k}\left\|D_{+t} d(\tau)\right\|_{x}^{2}+\sup _{t \geqslant s k}\left\|D_{+x} D_{+t} d(\tau)\right\|_{x}^{2}\right]\right\} \text {. }
\end{aligned}
$$

Adding (3.5), (3.12), (3.14) now proves the theorem. 
4. A Numerical Example. Several examples have been tested which confirm the results of Theorem 2.2, and we present one of them here.

Consider the wave equation written in the form

$$
\partial U / \partial t=\left[\begin{array}{cc}
0 & -1 \\
-1 & 0
\end{array}\right] \partial U / \partial x, \quad U=\left[\begin{array}{c}
U^{\mathrm{I}} \\
U^{\mathrm{II}}
\end{array}\right], \quad 0 \leqslant x \leqslant 1,
$$

with boundary conditions

$$
U^{\mathrm{I}}(0, t)=U^{\mathrm{I}}(1, t)=0 .
$$

It is approximated by the leap-frog scheme

$$
V_{\nu}(t+k)=V_{\nu}(t-k)-\alpha\left[\begin{array}{ll}
0 & 1 \\
1 & 0
\end{array}\right]\left(V_{\nu+1}(t)-V_{\nu-1}(t)\right), \quad \alpha=k / h,
$$

which is second-order accurate.

The boundary conditions are

$$
\left\{\begin{array}{l}
V_{0}^{\mathrm{I}}(t)=V_{N}^{\mathrm{I}}(t)=0, \\
V_{0}^{\mathrm{II}}(t)=2 V_{1}^{\mathrm{II}}(t)-V_{2}^{\mathrm{II}}(t), \\
V_{N}^{\mathrm{II}}(t)=2 V_{N-1}^{\mathrm{II}}(t)-V_{N-2}^{\mathrm{II}}(t) .
\end{array}\right.
$$

The last two conditions are locally second-order accurate, i.e., $\beta=m=2$ in (2.3c).

By a transformation of variables:

we obtain the scheme

$$
\begin{aligned}
y^{\mathrm{I}} & =\left(V^{\mathrm{I}}+V^{\mathrm{II}}\right) / \sqrt{2}, \\
y^{\mathrm{II}} & =\left(V^{\mathrm{II}}-V^{\mathrm{I}}\right) / \sqrt{2}, \quad y=\left(y^{\mathrm{I}}, y^{\mathrm{II}}\right)^{\prime},
\end{aligned}
$$

$$
y_{\nu}(t+k)=y_{\nu}(t-k)-\alpha\left(\begin{array}{cc}
-1 & 0 \\
0 & 1
\end{array}\right)\left(y_{\nu+1}(t)-y_{\nu-1}(t)\right)
$$

For the right half-plane problem, the boundary conditions are

$$
y_{0}^{\mathrm{I}}(t)=y_{0}^{\mathrm{II}}(t)
$$

$$
y_{0}^{\mathrm{I}}(t)+y_{0}^{\mathrm{II}}(t)=2\left(y_{1}^{\mathrm{I}}(t)+y_{1}^{\mathrm{II}}(t)\right)-\left(y_{2}^{\mathrm{I}}(t)+y_{2}^{\mathrm{II}}(t)\right) .
$$

The general solution in $l_{2}(0, \infty)$ to the resolvent equation is, for $|z|>1$,

where $\kappa_{1}$ satisfies

$$
\hat{w}_{\nu}=\left[\begin{array}{ll}
\lambda_{1} & \kappa_{1}^{\nu} \\
\lambda_{2} & \kappa_{2}^{\nu}
\end{array}\right],
$$

$$
z^{2} \kappa=\kappa+\alpha z\left(\kappa^{2}-1\right), \quad|\kappa|<1,
$$

and where $\kappa_{2}$ satisfies 


$$
z^{2} \kappa=\kappa-\alpha z\left(\kappa^{2}-1\right), \quad|\kappa|<1 .
$$

The boundary condition (4.6), with an inhomogenous term included, implies

$$
\left\{\begin{aligned}
\lambda_{1}-\lambda_{2} & =g_{0}^{(1)}, \\
\left(\kappa_{1}-1\right)^{2} \lambda_{1}+\left(\kappa_{2}-1\right)^{2} \lambda_{2} & =g_{0}^{(2)}
\end{aligned}\right.
$$

and the condition for a nontrivial solution is

$$
D(z) \equiv\left(\kappa_{1}-1\right)^{2}+\left(\kappa_{2}-1\right)^{2}=0 \text {. }
$$

Since $\kappa_{1}=-\kappa_{2},(4.9)$ means that $\kappa_{1}= \pm i$. These roots correspond to the $z$-values $z_{0}= \pm \alpha i \pm \sqrt{1-\alpha^{2}}$, and the determinant condition $\operatorname{Det}(D(1)) \neq 0$ of Theorem 2.2 is therefore satisfied.

It is easily shown that, in a neighborhood of $z_{0}$, there is a constant $c>0$ such that the determinant satisfies $|D(z)| \geqslant c\left|z-z_{0}\right|^{1 / 2}$. (The Eqs. (4.7a, b) have double roots at $z=z_{0}$.) From (4.8), it then follows that

$$
\left|\lambda_{1}\right|^{2}+\left|\lambda_{2}\right|^{2} \leqslant \frac{\text { const }}{\left|z-z_{0}\right|}\left(\left|g_{0}^{(1)}\right|^{2}+\left|g_{0}^{(2)}\right|^{2}\right)
$$

and we obtain

$$
\|\hat{w}\|_{x} \leqslant \frac{\text { const }}{(|z|-1)^{2}}\left|g_{0}\right|^{2} h
$$

in a neighborhood of $z=z_{0}$.

Since $\operatorname{Det}(D(z)) \neq 0$ at all other points $z$ with $|z| \geqslant 1$, the conditions of Theorem 2.2 are satisfied.

For the numerical experiment, the initial condition was

$$
\left.\begin{array}{l}
V_{\nu}^{\mathrm{I}}(0)=-\sin (2 \pi \nu / N) \\
V_{\nu}^{\mathrm{II}}(0)=0
\end{array}\right\}, \quad \nu=0,1, \cdots, N,
$$

and $V_{\nu}(k)$ was-obtained by the Lax-Wendroff one-step scheme. The scheme was run with $\alpha=0.9$ for $N=100,200,500$. The following table shows the error

$$
\|U(t)-V(t)\|_{x}=\left\|\left[\begin{array}{c}
-\cos (2 \pi t) \sin (2 \pi x) \\
\sin (2 \pi t) \cos (2 \pi x)
\end{array}\right]-V(t)\right\|_{x}
$$

at $t=0.45$ and $t=0.9$.

TABLE 4.1

\begin{tabular}{lll}
\hline & $t=0.45$ & $t=0.90$ \\
\hline$N=100$ & $1.40 \cdot 10^{-3}$ & $1.98 \cdot 10^{-3}$ \\
$N=200$ & $3.52 \cdot 10^{-4}$ & $4.96 \cdot 10^{-4}$ \\
$N=500$ & $5.63 \cdot 10^{-5}$ & $7.96 \cdot 10^{-5}$ \\
\hline
\end{tabular}


It is clear that the convergence rate is of second order.

Department of Computer Sciences

University of Uppsala

Uppsala, Sweden

1. B. GUSTAFSSON, H.-O. KREISS \& A. SUNDSTRÖM, "Stability theory of difference approximations for mixed initial boundary value problems. II," Math. Comp., v. 26, 1972, pp. 649686.

2. B. GUSTAFSSON, H.-O. KREISS \& A. SUNDSTRÖM, Stability Theory of Difference Approximations for Mixed Initial Boundary Value Problems. II, Dept. of Computer Sciences, Uppsala University, Report No. 30, 1970. 